Return to the Manage Active Submissions page at http://spie.org/submissions/tasks.aspx and approve or disapprove this submission. Your manuscript will not be published without this approval. Please contact author_help@spie.org with any questions or concerns.

\title{
Scheduling structural health monitoring activities for optimizing life- cycle costs and reliability of wind turbines
}

\author{
Anu Hanish Nithin \& Piotr Omenzetter \\ The LRF Centre for Safety and Reliability Engineering, The University of Aberdeen, Aberdeen, UK
}

\begin{abstract}
Optimization of the life-cycle costs and reliability of offshore wind turbines (OWTs) is an area of immense interest due to the widespread increase in wind power generation across the world. Most of the existing studies have used structural reliability and the Bayesian pre-posterior analysis for optimization. This paper proposes an extension to the previous approaches in a framework for probabilistic optimization of the total life-cycle costs and reliability of OWTs by combining the elements of structural reliability/risk analysis (SRA), the Bayesian pre-posterior analysis with optimization through a genetic algorithm (GA). The SRA techniques are adopted to compute the probabilities of damage occurrence and failure associated with the deterioration model. The probabilities are used in the decision tree and are updated using the Bayesian analysis. The output of this framework would determine the optimal structural health monitoring and maintenance schedules to be implemented during the life span of OWTs while maintaining a trade-off between the life-cycle costs and risk of the structural failure. Numerical illustrations with a generic deterioration model for one monitoring exercise in the life cycle of a system are demonstrated. Two case scenarios, namely to build initially an expensive and robust or a cheaper but more quickly deteriorating structures and to adopt expensive monitoring system, are presented to aid in the decision-making process.
\end{abstract}

Keywords: Decision tree analysis, pre-posterior Bayesian analysis, scheduling optimization, structural health monitoring, wind turbines

\section{INTRODUCTION}

The growing demands for wind energy and the need for reliable performance of Offshore Wind Turbines (OWTs) calls for increased research in the field of optimization of life-cycle costs and reliability of OWTs. Structural deterioration of many components of OWTs is a critical issue and the structures need to be inspected and maintained at regular intervals. The consequences of failure due to deterioration will have detrimental effects on the wind farm operations in terms of energy production and monetary and reputational losses. However, the operators are constantly faced with decision problems on choosing the optimal inspection or monitoring time intervals over the life-span of OWTs while reducing the operations and maintenance costs. Due to inaccessibility of OWTs during extreme weather, it is necessary to plan the inspections, monitoring and repair schedules in such a way that there is a good trade-off between the life-cycle costs and reliability of OWTs.

Optimization for structures such as bridges and oil and gas platforms has been carried out for cost, reliability, optimal operation and maintenance schedules using probabilistic methods [1-6]. Straub [7, 8] developed a detailed approach to risk based inspection methodology incorporating the Bayesian analysis and stochastic nature of deterioration models. Based on the risk-based inspection framework developed for oil and gas installations, similar work has been carried out for wind farms using the pre-posterior Bayesian decision analysis $[9,10]$. Maintenance optimization based on different conditions of degradation was performed for wind turbines (WT) blades where a Markov chain deterioration process has been adopted [11]. The costs associated with maintenance and primarily indirect costs, such as production losses and transportation costs, for wind power system have been minimized in [12].

This paper proposes to extend the existing frameworks for optimizing life-cycle costs to determine optimal monitoring schedules by incorporating the structural reliability/risk analysis (SRA) into the Bayesian pre-posterior analysis along with evolutionary optimization techniques such as genetic algorithms. Section 2 outlines the proposed framework for decision analysis for scheduling monitoring to optimize the life-cycle costs of OWTs. Section 3 presents a numerical illustration to compute the expected total cost from the decision tree analysis for one monitoring campaign executed during the life span of a structure. Two case scenarios are discussed to illustrate the decision-making process, namely 


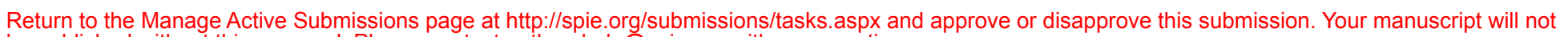
be published without this approval. Please contact author_help@spie.org with any questions or concerns.

whether to build initially a cheaper structure that will deteriorate at a faster rate and use an expensive monitoring system, or to build a robust but expensive structure and reduce the need for using expensive monitoring. Sections 4 and 5 round up the paper by presenting the results, conclusions and directions for future work.

\section{DECISION-MAKING MODEL}

A decision-making model is adopted herein to optimize the total expected life-cycle costs of wind turbines. The model comprises a structural deterioration model, a model for damage detection by an SHM system, and a cost model. All these are used in the pre-posterior decision analysis where the decisions need to be made to adopt or not adopt monitoring, the outcome of monitoring is detection or non-detection of damage, the decision rule is adopted to perform a repair action or not based on the SHM system indication, and the true state of the nature represents the actual state of the system. The analysis will be visualized with the help of a decision tree. The outcome of the decision tree analysis will determine the expected total life cycle cost including the cost of using a monitoring method. The different components of the decisionmaking model are outlined in the following subsections.

\subsection{Structural deterioration model}

A WT structure is assumed to be deteriorating over time $t$, in a process that is quantified with a generic damage intensity measure, $D$, that follows an exponential deterioration model [6]:

$$
\begin{aligned}
& D(t)=0 \quad \text { if } t<t_{0} \\
& D(t)=e^{\left(t-t_{0}\right) / \lambda}-1 \quad \text { if } t \geq t_{0}
\end{aligned}
$$

where $\lambda$ is the scale parameter and $t_{0}$ is the time to damage initiation in years. Methods such as the first order reliability methods (FORM) and simulation techniques such as Monte Carlo Simulations (MCSs) can be adopted to find the reliability profile of deteriorating structures based on the above Equations (1) and (2). MCS techniques using MATLAB [13] were adopted in this paper to calculate the probabilities of damage occurrence and failure due to deterioration.

Limit state functions (LSF) demarcate between the failure and safe domains of a structure. A typical LSF is denoted by $g(X)$, where $X$ is a vector of basic random variables, and the failure criterion is represented by $g(X) \leq 0$. A typical formulation of the probability of failure using an LSF is as follows:

$$
P(g(X) \leq 0)=\int_{g(X) \leq 0} f_{X}(x) d x
$$

where $g(X) \leq 0$ denote the failure domain of a probability density function, $f_{X}(x)$.

The time-dependent probability of damage occurrence, $P\left(D_{l}\right)$, is calculated from an LSF considering the occurrence of damage greater than a certain threshold, $D_{t h}$ :

$$
P\left(D_{1}\right)=P\left[D_{t h}-D(t) \leq 0\right]
$$

Note this implies that small damages, i.e. those for which $D(t)<D_{t h}$, are ignored. Similarly, the time-dependent probability of failure, $P_{f}(t)$, is calculated from another LSF by considering failure occurs when damage progresses to a greater intensity, beyond a failure threshold of $F_{t h}$ :

$$
P_{f}(t)=P\left[F_{t h}-D(t) \leq 0\right]
$$

The annual probability of failure, $P\left(F_{1}\right)$, for a time interval, $\Delta t$, is calculated from Equation (5):

$$
P\left(F_{1}\right)=\frac{P_{f}\left(t_{i}\right)-P_{f}\left(t_{i-1}\right)}{\Delta t\left(1-P_{f}\left(t_{i-1}\right)\right)}
$$

where $\Delta t_{i}$ refers to the time interval (1 year in our case). The above equation assumes that failure has not occurred before time $t_{i}[7]$. 


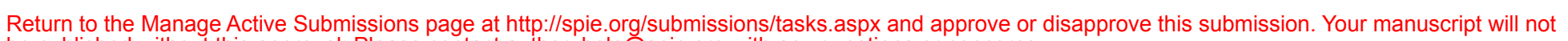
be published without this approval. Please contact author_help@spie.org with any questions or concerns.

The reliability index, $\beta$, of the structure with respect to the probability of failure can be approximated taking the value of the inverse Gaussian cumulative distribution function, $\Phi^{-1}$, at the probability of failure:

$$
\beta=-\Phi^{-1}\left(P\left(F_{1}\right)\right)
$$

where $\Phi^{-1}($.$) denotes the inverse Gaussian distribution function [14,15]$.

\subsection{Damage detection model}

The uncertainties associated with the ability of a monitoring technique to detect damage can be expressed using probabilistic approaches, e.g. using the probability of detection (POD) curves. The POD curves represent the probability of damage being detected by a monitoring method conditional on its extent (e.g. crack length or corrosion depth). POD is normally a monotonic curve that increases with the increase in damage intensity. The probability of minimum detectable damage is modeled herein using a cumulative lognormal probability distribution function, which is widely used for this purpose and can be expressed as [6]:

$$
P O D=\Phi\left(\frac{\ln (D)-\ln \alpha}{\beta}\right)
$$

where $\Phi($.$) is the standard normal cumulative distribution function, and \alpha$ and $\beta$ are the location and scale parameters, respectively, associated with the performance and quality of the monitoring method [16]. A comparison between POD curves of different hypothetical monitoring methods with different parameters $\alpha=0.1,0.3,0.5$ and $\beta=-0.1 \ln (\alpha)$ is presented in Figure 1. As seen from the figure, a monitoring method with a lower $\alpha$ has better quality as it detects smaller damage intensities.

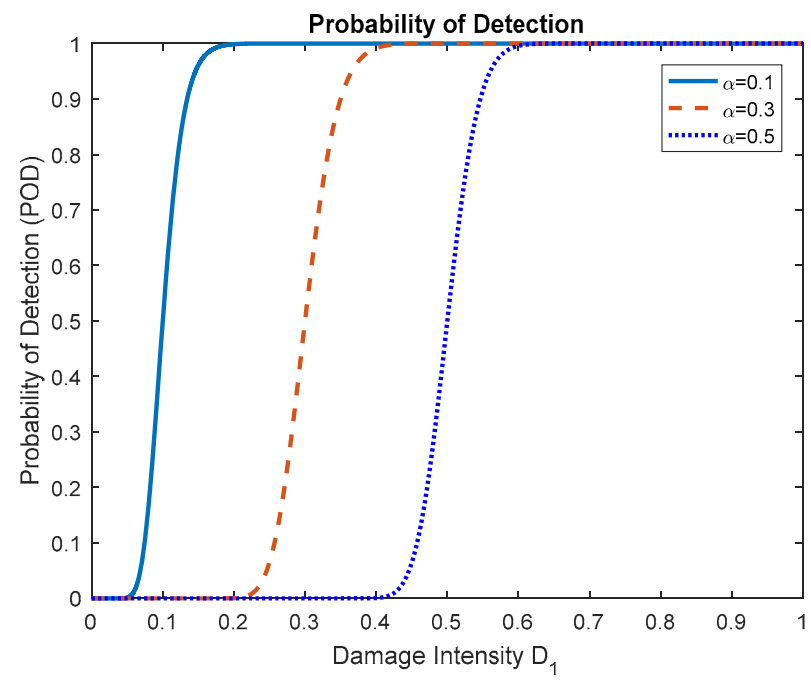

Figure 1. POD curves for different qualities of hypothetical monitoring methods

The incidence of false damage indications will arise for any monitoring method adopted. Events $D e_{0-1}$ represent the nonindication and indication of damage, respectively. The conditional probabilities of not indicating or indicating damage given the absence or presence of actual damage are expressed as $P\left(D e_{0-1} / D_{0-1}\right)$, see Table 1 . True positive values are the probability values of indicating damage when there exists actual damage. True negative values correspond to the probability of not indicating damage when there is no actual damage. False negative values correspond to Type 1 errors, i.e. when damage detection is missed when there exists actual damage. False positive values are Type II errors which arise from detection outcomes when there is no actual damage.

A graphical representation of the different scenarios of damage indication by a monitoring method is represented in Figure 2. A detection threshold is shown that acts as an acceptance criterion for damage detection such that the values to the right/left of the threshold will produce Type I/II errors [17, 18]. 
Return to the Manage Active Submissions page at http://spie.org/submissions/tasks.aspx and approve or disapprove this submission. Your manuscript will not be published without this approval. Please contact author_help@spie.org with any questions or concerns.

Table 1. True and false indications of damage by a monitoring method

\begin{tabular}{lll}
\hline & $\boldsymbol{D} \boldsymbol{e}_{\boldsymbol{0}}$ (no damage detection) & $\boldsymbol{D \boldsymbol { e } _ { \boldsymbol { 1 } } \text { (damage detection) }}$ \\
\hline $\boldsymbol{D}_{\boldsymbol{0}}$ (no damage present) & True Negative: $P\left(D e_{0} / D_{0}\right)$ & False Positive: $P\left(D e_{1} / D_{0}\right)$ \\
$\boldsymbol{D}_{\boldsymbol{1}}$ (damage present) & False Negative: $P\left(D e_{0} / D_{1}\right)$ & True Positive: $P\left(D e_{1} / D_{1}\right)$ \\
\hline
\end{tabular}

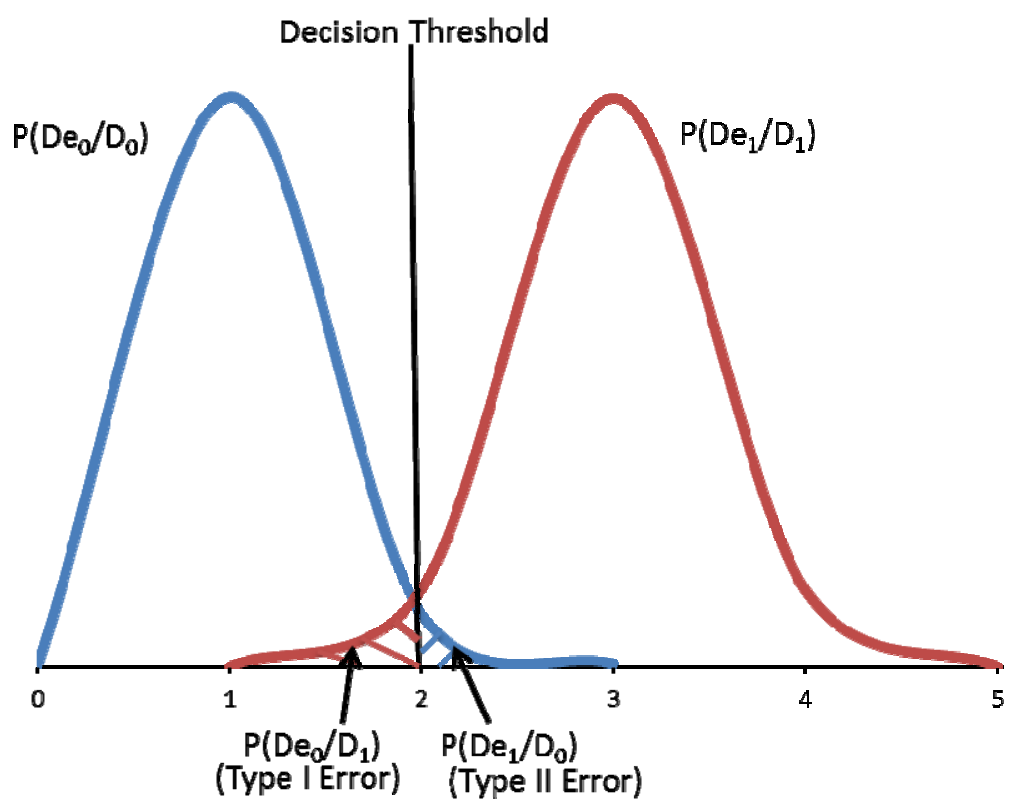

Figure 2. Theoretical representation of detection by a monitoring method

The probability of damage detection is obtained when the monitoring method detects damage intensities greater than $D_{d e}$, which is defined as the minimum detectable damage by the monitoring method. The minimum detectable damage is modeled by the lognormal POD function as defined in Equation (8) [19]:

$$
P\left(D e_{1}\right)=P\left(D_{d e}-D(t) \leq 0\right)
$$

The true positive values of monitoring method, $P\left(D e_{1} / D_{1}\right)$, which is also the conditional probability of the event of the damage detection given the occurrence of damage is calculated as:

$$
P\left(D e_{1} / D_{1}\right)=\frac{P\left(D e_{1} \cap D_{1}\right)}{P\left(D_{1}\right)}
$$

The numerator of Equation (10) is calculated by considering the events $D e_{1}$ and $D_{1}$ to be in a parallel system and the system probability, $P_{s}$, is represented in Equation (11).

$$
P_{s}=P\left(\bigcap_{i=1}^{n}\left\{g_{i}(\mathrm{X}) \leq 0\right\}\right)
$$

where $g_{i}(X)$ represents the limit state function of $n$ failure events and is calculated by using the simple bounds as given in Equation (12) [20]: 
Return to the Manage Active Submissions page at http://spie.org/submissions/tasks.aspx and approve or disapprove this submission. Your manuscript will not be published without this approval. Please contact author_help@spie.org with any questions or concerns.

$$
0 \leq P_{s} \leq \min \left(P\left(D_{t h}-D(t) \leq 0\right), P\left(D_{d e}-D(t) \leq 0\right)\right)
$$

An average of the lower and upper bounds is considered from the above simple bounds. It should be noted that the simple bounds are practically wide and second-order bounds should be considered for accurate calculation of probabilities. The probabilities outlined in Table 1 are computed in an equivalent manner.

\subsection{Cost model}

The total costs in each branch of the decision tree will comprise of the cost of initial construction, failure, monitoring and repair. This cost in each branch is calculated using the discounted rate of money, $r$. At any time monitoring is undertaken, $t_{\text {monit }}$, the total discounted costs can be calculated as [4]:

$$
C_{B 1-B 18}=\frac{C_{\text {initial }, i}}{(1+r)^{t_{\text {intital }}}}+\frac{C_{\text {monit }, i}+C_{r, i} \times x_{t, i}+C_{f, i} \times y_{t, i}}{(1+r)^{t_{\text {monit }, i}}}
$$

where $C_{\text {initial }}$ is the cost of initial construction at $t_{\text {initial }}=0 ; C_{\text {monit }}$ is the cost of a monitoring campaign $\left(C_{\text {monit }}=0\right.$ when no monitoring/inspection is performed), $C_{r}$ is the cost of repair, $C_{f}$, is the cost of failure, and $r$ is the annual discount rate of money. Binary variables $x$ and $y$ represent the occurrence of repair and failure event in the decision tree branch:

$$
\begin{aligned}
& x= \begin{cases}1 & \text { if repair action appears in the decision tree branch } \\
0 & \text { otherwise }\end{cases} \\
& y= \begin{cases}1 & \text { if failure occurs in the decision tree branch } \\
0 & \text { otherwise }\end{cases}
\end{aligned}
$$

\subsection{Decision tree analysis}

The probabilities calculated from the deterioration and damage detection models are used in the construction of a decision tree to determine the optimal monitoring time(s) during the life-cycle of the wind turbine. Let $M_{0}$ and $M_{I}$ represent the decision to not adopt a monitoring method and adopt it, respectively, $D e_{0}$ and $D e_{1}$ be, as before, the event of not indicating and indicating a damage (corresponding to an outcome of monitoring), $R_{0}$ and $R_{l}$ represent the decision to not repair or repair (corresponding to a decision rule), $D_{0}$ and $D_{l}$ denote no occurrence or occurrence of actual damage (corresponding to the true state of nature), and $F_{0}$ and $F_{l}$ denote no failure or failure of the structure (corresponding to the true state of nature). A decision tree formulated for adopting one monitoring campaign is presented in Figure 3.

A decision is made to employ $\left(M_{1}\right)$ or not employ $\left(M_{0}\right)$ a monitoring system. In the top branch, when monitoring is not adopted $\left(M_{0}\right)$, the repair actions are performed based on the prior probabilities of damage occurrence. The random outcomes or states of nature are the occurrence of the damage and further consequences such as failure of the structure are represented in Nodes 1-2. In the alternative lower branch corresponding to adopting a monitoring scheme $\left(M_{l}\right)$, the decision will lead to a monitoring detection outcome (Node 10) that may subsequently lead to performing repair actions (Node 6 and 9). The true states of nature (damage and failure) are represented in Nodes 4-5 and 7-8. The posterior probabilities of damage and failure given the detection or non-detection are updated using the Bayes rule [21] in this branch. In the event of damage detection, the updated probability of damage indication is given as follows [22]:

$$
P_{D e_{1}, \text { updated }}=P\left(g_{i}(\mathrm{X}) \leq 0 / D e_{1} \leq 0\right)=\frac{P\left(g_{i}(\mathrm{X}) \leq 0 \cap D e_{1} \leq 0\right)}{P\left(D e_{1} \leq 0\right)}
$$

In the event of non-detection of damage, the updated probability is given as follows:

$$
P_{D e_{0}, \text { updated }}=P\left(g_{i}(\mathrm{X}) \leq 0 / D e_{0} \leq 0\right)=\frac{\left(P\left(g_{i}(\mathrm{X}) \leq 0\right)\right)-\left(P\left(g_{i}(\mathrm{X}) \leq 0 / D e_{1} \leq 0\right) P\left(D e_{1} \leq 0\right)\right)}{1-P\left(D e_{1} \leq 0\right)}
$$


Return to the Manage Active Submissions page at http://spie.org/submissions/tasks.aspx and approve or disapprove this submission. Your manuscript will not be published without this approval. Please contact author_help@spie.org with any questions or concerns.

where $g_{i}(\mathrm{X})$ represents the limit state functions of events of damage occurrence and failure for each branch as outlined in the decision tree. The costs corresponding to each branch are denoted by $C_{B I-B I 8}$ as explained by the cost model in Section 2.3. The expected cost at each chance node is the sum of the products of the respective probabilities and the costs in each branch. The expected cost selected at each decision node is the minimum value of the expected costs coming into that decision node. Using the rollback technique [23], the expected total cost following the decision of installing a monitoring system can be determined using this model. The total expected cost of failure over the design life, $T_{D L}$, is added to the total expected cost calculated from the decision tree [7]:

$$
\mathrm{E}\left[C_{F}\right]=\sum_{t_{i}=1}^{T_{D L}} \frac{C_{f, i} P\left(F_{1}\right)\left(1-P_{f}\left(t_{i-1}\right)\right)}{(1+r)^{t_{i}}} \Delta t_{i}
$$

The quantification of the value any monitoring method can be achieved by estimating the Value of Information (VOI) of the monitoring method. Through the determination of VOI, the benefits of performing an experiment, such as performing a monitoring campaign, can be calculated. The VOI can be computed by considering the difference of the total expected costs under no-monitoring scenario and the total expected costs under monitoring scenario [24].

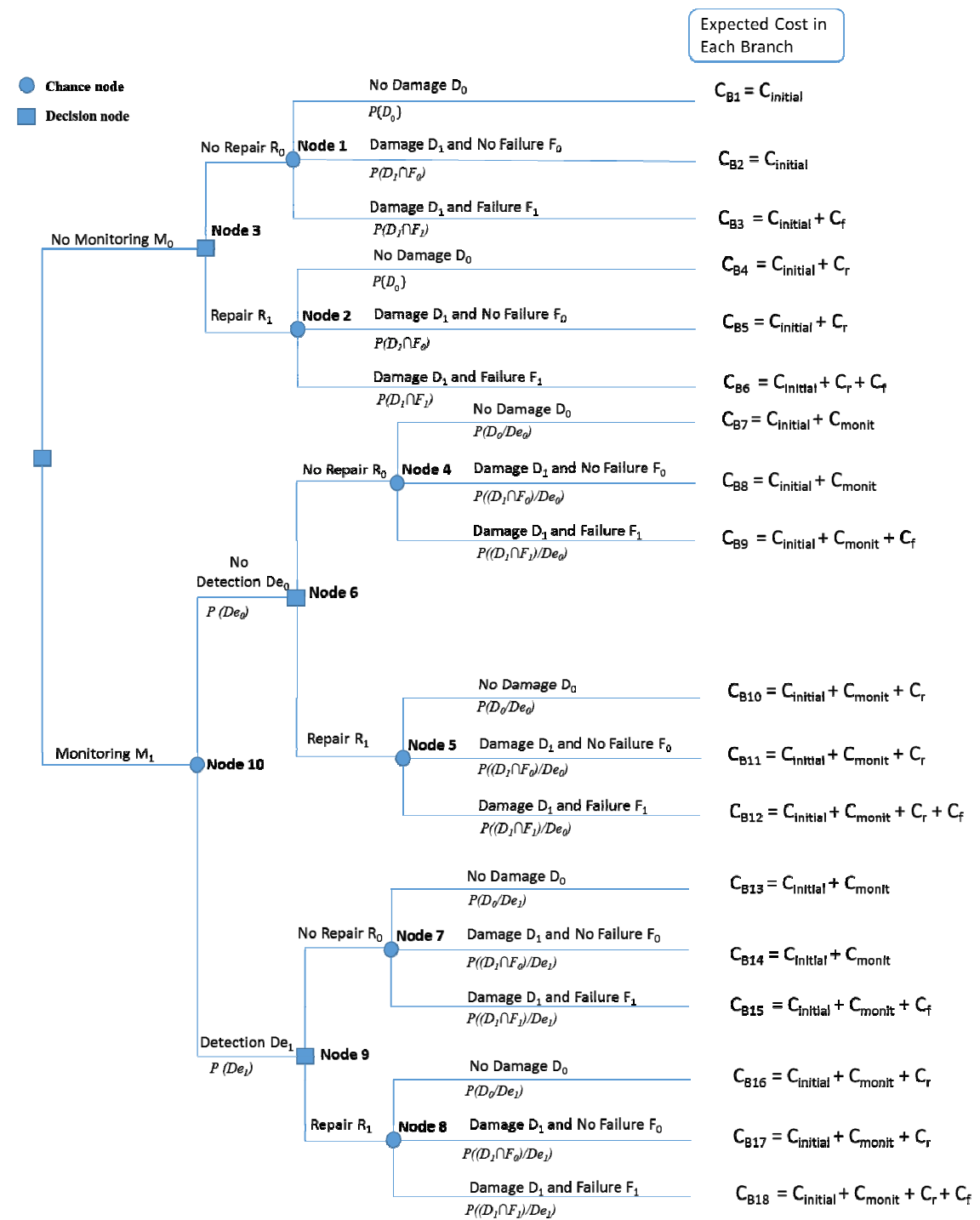

Figure 3. Decision tree for a single monitoring campaign 
Return to the Manage Active Submissions page at http://spie.org/submissions/tasks.aspx and approve or disapprove this submission. Your manuscript will not be published without this approval. Please contact author_help@spie.org with any questions or concerns.

\section{NUMERICAL ILLUSTRATIONS}

\subsection{A single monitoring campaign over the life-span}

For illustrative purposes, an example of the generic framework that can be applied on any structural component of a WT to find the optimal monitoring time with an assumption of adopting one monitoring schedule over the entire design life is provided in this section. A design life of 20 years for the structure is assumed. The input parameters for the deterioration model, described in Equations (1) and (2), are $\lambda$, which follows a lognormal distribution with a mean $(\mu)$ of 50/years and standard deviation $(\sigma)$ of 10/years, and $t_{0}$, which follows a lognormal distribution with a mean and standard deviation of 3 years and 1 year, respectively. The thresholds for damage occurrence, $D_{t h}$, and failure, $F_{t h}$, are assumed to be 0.1 and 0.3 , respectively. The probabilities of damage occurrence and failure and the reliability indices of the structure over its entire assumed life are calculated from Equations (4), (5) and (7) through MCSs with 100,000 samples and are presented in Figure 4.
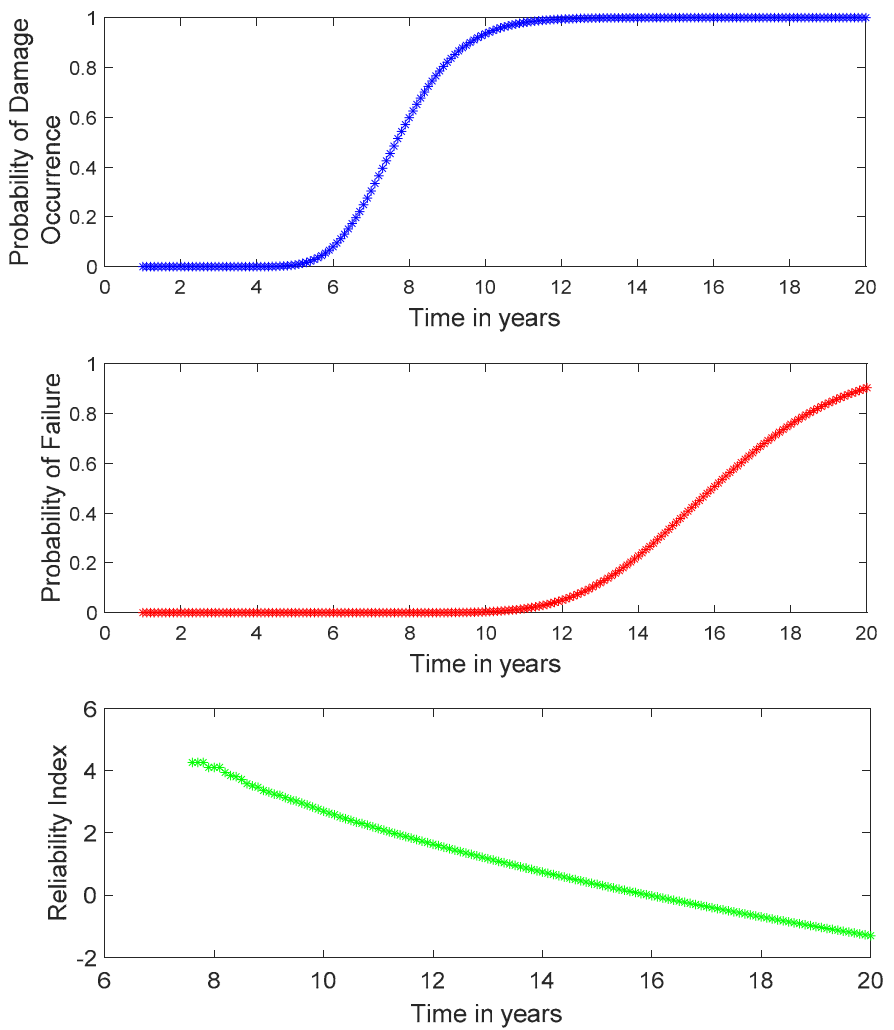

Figure 4. Probability of damage occurrence, failure and reliability index over time

The intersection probabilities of the event of damage occurrence and failure or no-failure of the structure are computed using the probabilities of a parallel system as outlined in Section 2.2 and are presented in Figure 5.
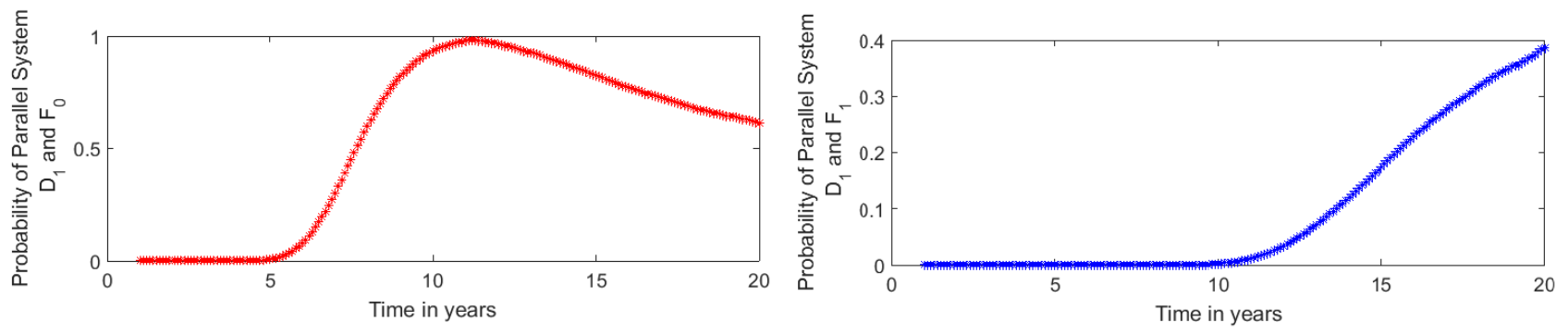

Figure 5. Probability of parallel failure events $\mathrm{P}\left(D_{l} \cap F_{0}\right)$ and $\mathrm{P}\left(D_{l} \cap F_{l}\right)$ 
Return to the Manage Active Submissions page at http://spie.org/submissions/tasks.aspx and approve or disapprove this submission. Your manuscript will not be published without this approval. Please contact author_help@spie.org with any questions or concerns.

The probability of damage detection, $P\left(D e_{1}\right)$, is computed as outlined in Section 2.2 and is presented in Figure 6 .

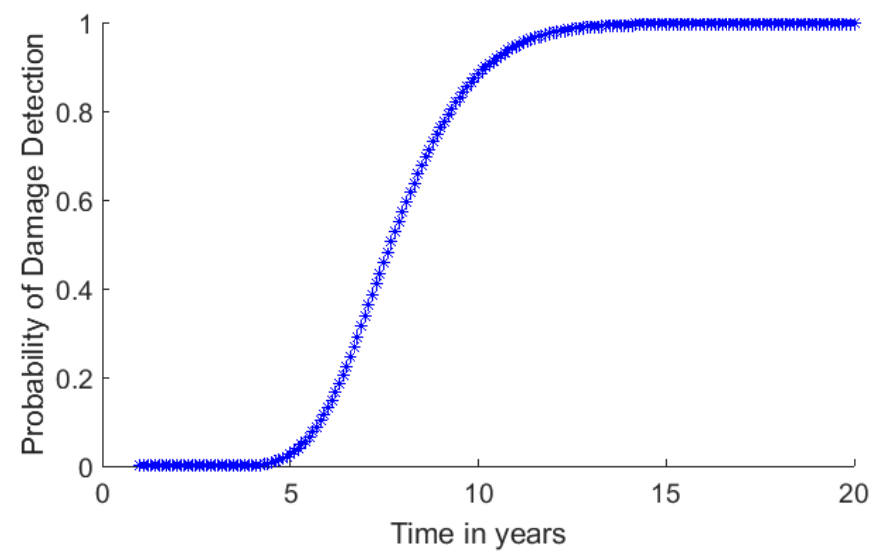

Figure 6. Probability of damage detection $P\left(D e_{1}\right)$

The assumed representative costs are $C_{\text {initial }}=£ 100,000, C_{\text {monit }}=£ 1,000$ for a monitoring system with $\alpha=0.1, C_{r}=£ 1,000, C_{f}$ $=£ 100,000$. The discount rate of money, $r$, is assumed to be $3.5 \%$. Using the decision tree presented in Figure 3 and Equation (13), the total expected life-cycle cost of the structure is calculated using the rollback technique as explained in Section 2.3. The results of total expected life-cycle cost under no-monitoring and monitoring scenario with respect to the time of monitoring or repair are presented in Figure 7. As seen in Figure 7, a minimum value of the total expected cost of $£ 97,000$ under the monitoring scenario is obtained which determines the optimal monitoring time after 6.4 years over the service life of 20 years using the proposed decision-making model as described in Section 2. The benefits of having a monitoring method is computed which gives a VOI of $£ 55,000$ at 6.4 years.

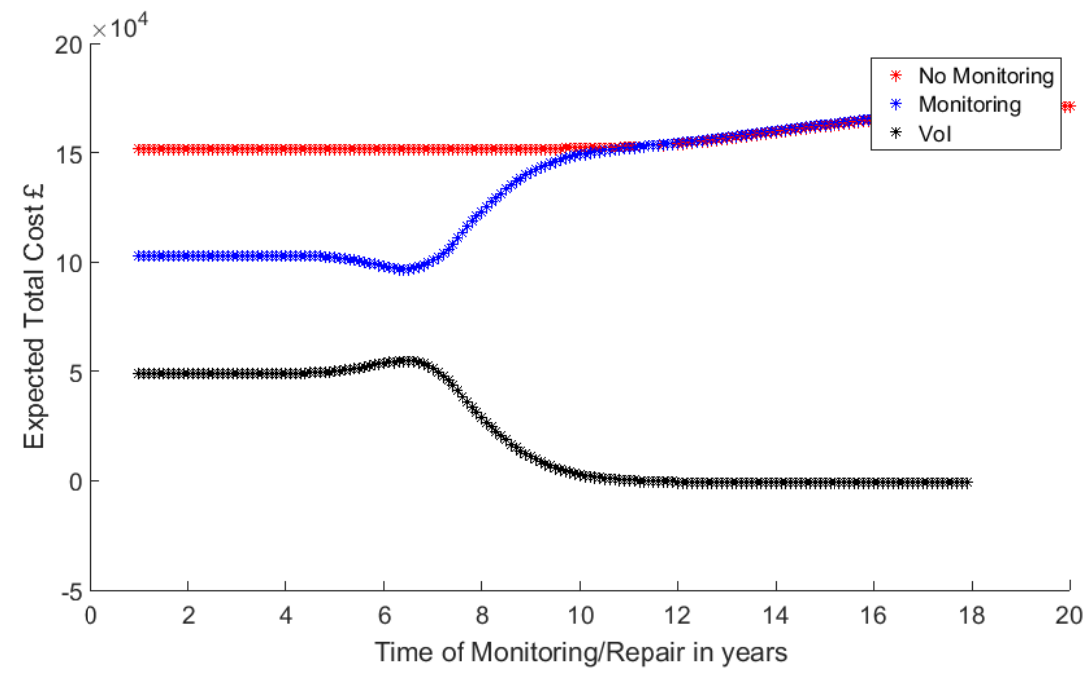

Figure 7. Expected total cost and VOI for a single monitoring campaign

\subsection{Two case scenarios}

In another illustrative example, two scenarios are considered to explore the proposed model. The first scenario (Case I) proposes designing and constructing a cheaper structure which may, however, be subjected to early deterioration. To counteract this, a high quality but expensive system of monitoring will be considered in this instance. The second scenario (Case II) proposes building a robust, durable but expensive structure and adopting a cheaper monitoring system which, however, entails lower quality of monitoring. The design life of 20 years is considered for these two cases. The 
Return to the Manage Active Submissions page at http://spie.org/submissions/tasks.aspx and approve or disapprove this submission. Your manuscript will not be published without this approval. Please contact author_help@spie.org with any questions or concerns.

input parameters and the representative costs are presented in Table 2. The comparison between the probabilities of damage occurrence, failure and reliability indices for the two scenarios are presented in Figure 8.
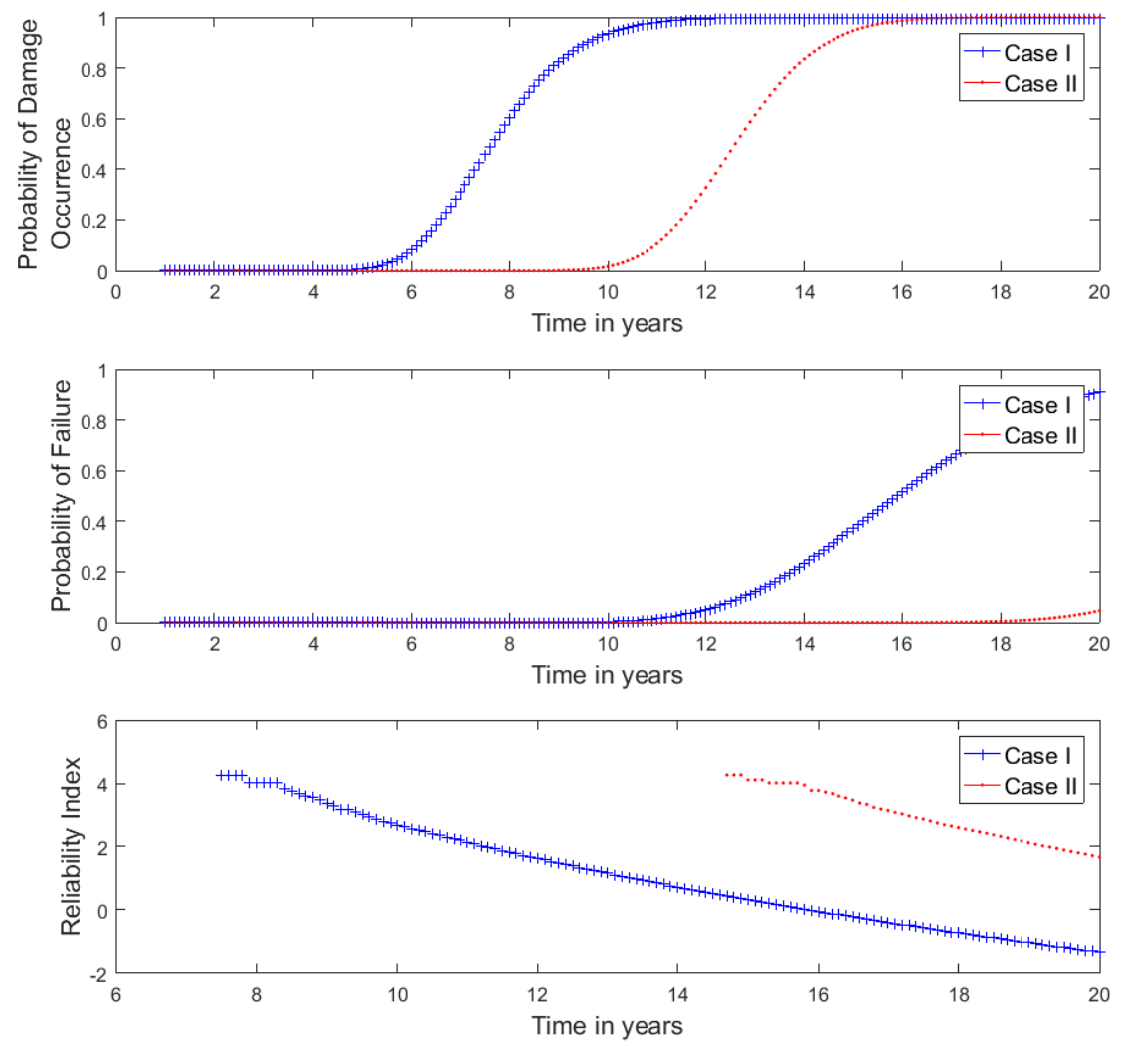

Figure 8. Comparison between time-dependent probabilities of damage occurrence and failure for two cases

Table 2. Input parameters for two case scenarios

\begin{tabular}{lll}
\hline Input parameters & Case I & Case II \\
\hline$\lambda$ (lognormal distribution) & $\mu=50 /$ year; $\sigma=10 /$ year & $\mu=70 /$ year; $\sigma=10 /$ year \\
$t_{0}$ (lognormal distribution) & $\mu=3$ years; $\sigma=1$ year & $\mu=6$ years; $\sigma=1$ year \\
$\alpha$ & 0.1 & 0.5 \\
$C_{\text {initial }}$ & $£ 100,000$ & $£ 120,000$ \\
$C_{\text {monit }}$ & $£ 1,000$ & $£ 500$ \\
$C_{r}$ & $£ 10,000$ & $£ 10,000$ \\
$C_{f}$ & $£ 100,000$ & $£ 1,000,000$ \\
\hline
\end{tabular}

The total expected life-cycle costs for the two cases calculated from the decision tree using the rollback technique are provided in Figure 9. A comparison of expected total costs for the two cases when monitoring or no-monitoring techniques are adopted are presented along with the VOI. The expected cost under no-monitoring scenario is calculated at Node 3 in the decision tree as shown in Figure 3. This cost is dependent on the cost of failure and repair. The prior probabilities of damage occurrence and failure are considered for no-monitoring scenario over the design life of 20 years.

The expected cost under the monitoring scenario is determined at Node 10 in the decision tree as shown in Figure 3. This cost also accounts for the cost of monitoring in addition to the cost of failure and repair. The probabilities of damage occurrence are updated based on monitoring outcome of detection or no-detection using Bayes rule as provided in Section 2.4. These updated posterior probabilities of the damage occurrence and failure or no-failure consequence are used for the determination of the total expected costs in this scenario. The initial capital costs are included in both 
Return to the Manage Active Submissions page at http://spie.org/submissions/tasks.aspx and approve or disapprove this submission. Your manuscript will not be published without this approval. Please contact author_help@spie.org with any questions or concerns.

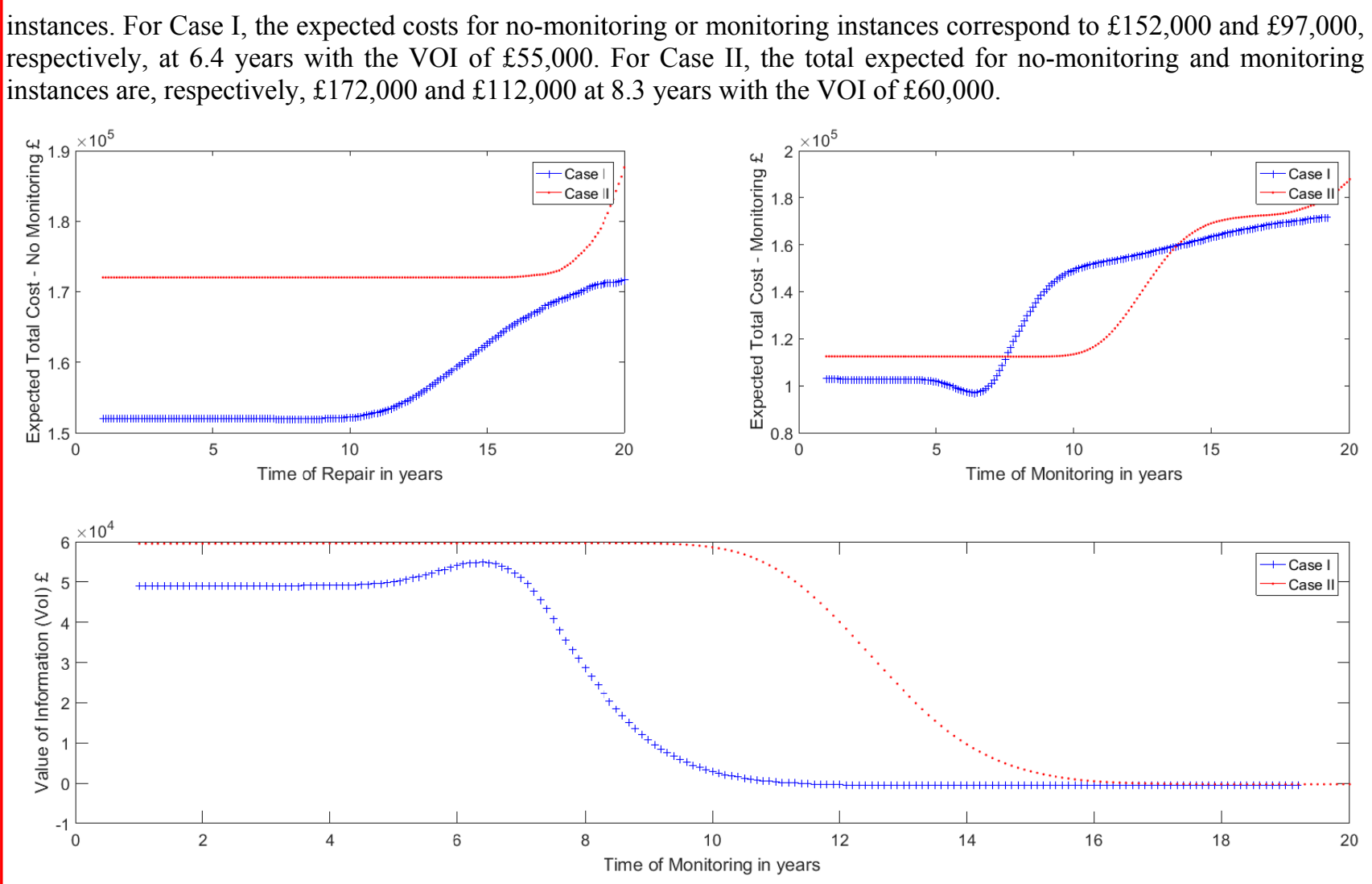

Figure 9. Expected total life-cycle costs and VOI for two cases

\section{RESULTS}

The optimal monitoring campaign times determined by using the proposed framework have been outlined in Table 3. It should be noted that the models proposed are generic to any structure and the optimal monitoring times will vary as per the specific input models used for a WT structure such as deterioration models specific to, e.g., fatigue or corrosion. However, practical applications could follow the same methodology of the proposed decision-making framework as described in Section 2.

Table 3. Optimal monitoring times over the design life of the structure

\begin{tabular}{|c|c|c|c|}
\hline Case scenario & $\begin{array}{l}\text { Optimal } \\
\text { inspection } \\
\text { time (years) }\end{array}$ & $\begin{array}{l}\text { Expected total } \\
\text { cost }(£)- \\
\text { Monitoring }\end{array}$ & VOI $€$ \\
\hline Case I (Single inspection) & 6.4 & 97,000 & 55,000 \\
\hline Case II (Single inspection) & 8.3 & 112,000 & 60,000 \\
\hline
\end{tabular}

\section{CONCLUSIONS AND FUTURE WORK}

A decision-making framework to determine the optimal monitoring campaign times over the design life of a WT structure has been developed. The framework integrates structural reliability/risk analysis with the Bayesian preposterior analysis along with decision tree models and has been illustrated with several generic examples. The future work will comprise extending the framework with formal optimization techniques such as genetic algorithms. The output of the optimization algorithms will provide a set of optimal solutions that provide a trade-off between the life-cycle costs 
Return to the Manage Active Submissions page at http://spie.org/submissions/tasks.aspx and approve or disapprove this submission. Your manuscript will not be published without this approval. Please contact author_help@spie.org with any questions or concerns.

and reliability of the WT structure that would determine the optimal monitoring times. This will be further illustrated by considering a specific deterioration model such as fatigue on a component of the WT.

\section{AKNOWLEDGEMENT}

Anu Hanish Nithin's PhD study within the Lloyd's Register Foundation Centre for Safety and Reliability Engineering at the University of Aberdeen is supported by Lloyd's Register Foundation. The Foundation helps to protect life and property by supporting engineering-related education, public engagement and the application of research.

\section{REFERENCES}

[1] Frangopol, D.M. and Soliman, M., (2016). Life-cycle of structural systems: recent achievements and future directions, Structure and Infrastructure Engineering, 12 (1), pp.1-20.

[2] Barone, G. and Frangopol, D.M., (2014). Life-cycle maintenance of deteriorating structures by multi-objective optimization involving reliability, risk, availability, hazard and cost, Structural Safety, 48 pp.40-50.

[3] Dong, Y. and Frangopol, D.M., (2015). Risk-informed life-cycle optimum inspection and maintenance of ship structures considering corrosion and fatigue, Ocean Engineering, 101 pp.161-171.

[4] Frangopol, D., Lin, K. and Estes, A., (1997). Life-cycle cost design of deteriorating structures, Journal of Structural Engineering, 123 (10), pp.1390-1401.

[5] Frangopol, D.M. and Maute, K., (2003). Life-cycle reliability-based optimization of civil and aerospace structures, Computers \& Structures, 81 (7), pp.397-410.

[6] Kim, S., Frangopol, D. and Soliman, M., (2013). Generalized probabilistic framework for optimum inspection and maintenance planning, Journal of Structural Engineering, 139 (3), pp.435-447.

[7] Straub, D., (2004). Generic approaches to risk based inspection planning for steel structures, Doctor of Technical Sciences. Swiss Federal Institute of Technology Zurich

[8] Straub, D. and Faber, M.H., (2005). Risk based inspection planning for structural systems, Structural Safety, 27 (4), pp.335-355.

[9] Sorensen, J.D., (2009). Framework for risk-based planning of operation and maintenance for offshore wind turbines, Wind Energy, 12 (5), pp.493-506.

[10] Nielsen, J.J. and Sorensen, J.D., (2011). On risk-based operation and maintenance of offshore wind turbine components, Reliability Engineering \& System Safety, 96 (1), pp.218-229.

[11] F. Besnard and L. Bertling, (2010). An approach for condition-based maintenance optimization applied to wind turbine blades, IEEE Transactions on Sustainable Energy, 1 (2), pp.77-83.

[12] F. Besnard, M. Patrikssont, A. B. Strombergt, A. Wojciechowskit and L. Bertling, (2009). An optimization framework for opportunistic maintenance of offshore wind power system, PowerTech, 2009 IEEE Bucharest, pp. $1-7$.

[13] The MathWorks, Inc., Natick, Massachusetts, United States., Matlab, R2016b

[14] Melchers, R.E., (1987). Structural Reliability: Analysis and Prediction. Chichester, W.Sussex,England: Wiley.

[15] Madsen, H.O., Krenk, S. and Lind, N., (1986). Methods of Structural Safety. Prentice-Hall.

[16] Forsyth, D. and Fahr, A., (1998). An evaluation of probability of detection statistics, Institute of Aerospace Research, pp.10-1.

[17] Matzkanin, G. and Yolken, H., (2001). A technology assessment of probability of detection (POD) for nondestructive evaluation (NDE), (NTIAC-TA-00-01),

[18] Omenzetter, P., Limongelli, M. and Yazgan, U., (2016). A pre-posterior analysis framework for quantifying the value of seismic monitoring and inspections of buildings, Report No. COST Action TU1402: Quantifying the Value of Structural Health Monitoring,

[19] Sorensen, J., Faber, M., Rackwitz, R. and Thoft-Christensen, P., (1991). Modelling in optimal inspection and repair, OMAE 91 Conference, Stavanger, Norway, pp. 281-288.

[20] Sorensen, J.D., (2004). Notes in Structural Reliability Theory and Risk Analysis, Available at: http://www.tbm.tudelft.nl/fileadmin/Faculteit/CiTG/Over_de faculteit/Afdelingen/Afdeling_Waterbouwkunde/sect ie waterbouwkunde/people/personal/gelder/publications/citations/doc/citatie215.pdf

[21] Walpole, R., Myers, R., Myers, S. and Ye, K., (2014). Probability and Statistics for Engineers and Scientists. USA: Pearson New International Edition.

[22] Eltaief, M., Bouraoui, C. and Chateauneuf, A., (2013). Inspection time optimization for a cracked component based on a reliability approach, In Haddar, M., Romdhane, L., Louati, J. and Ben Amara, A., (Eds.) Design and Modeling 


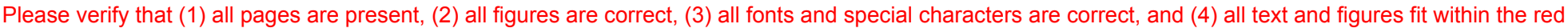
margin lines shown on this review document. Complete formatting information is available at http://SPIE.org/manuscripts

Return to the Manage Active Submissions page at http://spie.org/submissions/tasks.aspx and approve or disapprove this submission. Your manuscript will not be published without this approval. Please contact author_help@spie.org with any questions or concerns.

of Mechanical Systems: Proceedings of the Fifth International Conference Design and Modeling of Mechanical Systems, CMSMÂ'2013, Djerba, Tunisia, March 25-27, 2013Berlin, Heidelberg: Springer Berlin Heidelberg, pp. 405-413.

[23] Magee, J., (1964). Decision Trees for Decision Making, Available at: https://hbr.org/1964/07/decision-trees-fordecision-making

[24] Raiffa, H. and Schlaifer, R., (1961). Applied Statistical Decision Theory. Boston: Harward University. 